\title{
Influence of microstructure on the transitions between mesoscopic thin-film morphologies in ballistic-diffusive models
}

\author{
Pedro A. Sánchez, ${ }^{1, *}$ Tomás Sintes, ${ }^{1, \dagger}$ Julyan H. E. Cartwright, ${ }^{2, \ddagger}$ and Oreste Piro ${ }^{1, \S}$ \\ ${ }^{1}$ Depto. de Física, Instituto de Física Interdisciplinar y Sistemas Complejos (IFISC), CSIC-UIB, \\ Universitat de les Illes Balears, E-07122 Palma de Mallorca, Spain \\ ${ }^{2}$ Instituto Andaluz de Ciencias de la Tierra (IACT), CSIC-UGR, Campus Fuentenueva, E-18002 Granada, Spain
}

(Received 31 July 2009; published 29 January 2010)

\begin{abstract}
We study the influence of the symmetries of competing microstructures on the emergence of different mesoscopic morphologies in the growth by vapor deposition of thin solid films. We show the results of numerical simulations in $(1+1)$ - and (2+1)-dimensional systems including different microstructures, as well as thermally activated surface diffusion in combination with a ballistic algorithm to model the deposition process. We focus on the characterization of the transitional structures that appear in the empirical structure zone model (SZM) through the evaluation of the mean packing density and the mean coordination number. We show that the maximum coordination number of the underlying microstructure classifies the statistics of the transitional morphologies at the border between zone I in the SZM, characterized by the formation of fractal-like patterns, and zone II, where pronounced faceting develops. We analyze the appearance of lattice frustration and texture competition effects in complex microstructures having mutually exclusive symmetries.
\end{abstract}

DOI: 10.1103/PhysRevE.81.011140

PACS number(s): 05.70.Fh, 81.15.Aa, 68.55.-a, 05.10.Ln

\section{INTRODUCTION}

The growth of thin solid films by different deposition techniques has been an active field of research in the past decades driven by the variety and increasing relevance of technological applications such as those in microelectronics, optics, chemistry, and biology $[1,2]$. On the other hand, from a theoretical perspective, the dynamics of thin-film growth exhibits very interesting and challenging problems related to key topics in nonlinear and statistical physics such as critical phenomena and universality. Not surprisingly, the modeling of film growth has developed in parallel with advances in the study of growth processes far from equilibrium, fractal growth, and interface physics $[3,4]$.

One of the main difficulties in studying the growth of thin films is the wide range of length and time scales involved during film formation which, in practice, require the adoption of different approaches for each scale domain. The most recent modeling efforts focus on the integration of different techniques within large multiscale frameworks [5-7]. Nevertheless, single-scale techniques are still widely used in order to study specific aspects of the growth dynamics. For instance, electronic interactions and atomic vibrations, which take place in length and time scales typically below $10^{-6} \mathrm{~m}$ and $10^{-10} \mathrm{~s}$, respectively, are presently studied through molecular-dynamics simulations, in which density-functional theory and empirical or semiempirical potentials accounting for the atomic mobility processes, such as thermally activated diffusive hops, are widely used in order to predict the film microstructures. On the other hand, a variety of continuum models have been proposed to study mesoscopic and

\footnotetext{
*pedro@ifisc.uib.es

†tomas@ifisc.uib.es

¥ulyan.cartwright@csic.es

\$piro@ifisc.uib.es
}

macroscopic properties of the epitaxial thin-film growth in which the interface is described by a continuous height function [8]. More recently, nonlinear differential equations have been derived for the surface shape evolution from the transport equations for the adatoms in the micron or even nanometer scale $[9,10]$. Such analytical studies are often compared with kinetic Monte Carlo (kMC) simulations. Typically, and for intermediate scales, the most frequently used models are based on atomistic simulations that exclude the details of atomic vibrations and concentrate on simulating the atomic mobility processes as stochastic events via Monte Carlo methods.

The simplest atomistic stochastic models adopt drastic approximations to the deposition and growth processes in order to achieve computational efficiency. In this approach, particles-atoms, molecules, or clusters, sometimes called growth units or adatoms - are deposited onto a substrate via ballistic trajectories. The interactions are limited to first nearest neighbors and, in many cases, simulations are in $(1+1)$ dimensions. For crystalline films, simple regular latticestypically square or hexagonal-are used, while amorphous materials are commonly modeled through off-lattice simulations without any bonding symmetry restriction.

Despite—or arguably owing to-these approximations, simple atomistic models have proved a suitable tool for studying different aspects of thin solid film growth regimes and morphologies. These models allow for the control of certain growth parameters such as the shadowing of lower film surface regions by prominent structures and the change in deposition angles with respect to the substrate [11-15]. Within this modeling framework, special attention has been paid in the past to the possible universality of the kinetic roughening of the growing film surface [4,16-22]. On the contrary, the interplay between film bulk properties and the surface evolution [23] has not been intensively studied with this type of models, probably because of the assumption that the bulk properties should depend strongly on the micro- 
scopic details of the atomic interactions that are not included in simple mesoscopic or continuum models.

Nevertheless, one of the most universal behaviors of film growth observed experimentally is the existence of diverse characteristic mesoscopic morphologies, with different surface and bulk properties, arising as a consequence of the competition between the spatially disordered deposition of particles on the growing film and the ordering effect due to mobility processes. These characteristic morphologies have been summarized in successive qualitative empirical models known as structure zone models (SZM). The first SZM was introduced by Movchan and Demchishin [24] for physical vapor deposition under simple experimental conditions. They characterized three morphologically different regions obtained by varying the deposition rate and the substrate temperature. Progressively, new structure zones have been incorporated into the original model as different experimental parameters have been introduced, such as the activated surface diffusion by ion bombardment of the film surface, the presence of an inert gas in the deposition chamber, the effects of chemical absorption-desorption processes [25,26], the presence of impurities [27], etc. The reproduction of the diverse structure zones and their dependence on the different deposition parameters has become a test for simple atomistic models. However, in spite of this great progress, little effort has been devoted to the characterization of the transitional structures at parameters near the zone boundaries. In particular, the impact of the symmetries of the microstructure on the nature of these transitional structures has not been fully analyzed either experimentally or theoretically.

The main purpose of this paper is to go beyond qualitative description of the similarities observed within each structure zone and to investigate by means of simulations the influence of the material microstructure symmetries on the transitional mesoscale morphologies of the grown film. For this purpose, we have developed a minimal stochastic ballistic deposition model with thermally activated surface diffusion in $(1+1)$ and $(2+1)$ dimensions to study the physics of solid film growth operating at the interface between the molecular scale and the mesoscale.

Our simulations show that the statistical features of the transitional film morphologies obtained when the parameters change from the so-called zone I in the SZM, characterized by the formation of fractal-like patterns, to zone II, where pronounced faceting emerges with the posterior formation of characteristic smooth surfaces, depend on the number of active positions compatible with the substrate orientation that is reflected via the maximum coordination number of the underlying microstructure. We also show that distinct microstructures have clearly discernable effects on the evolution of the characteristic sizes of compact mesoscopic structures that compose the films in zone II. These results demonstrate the relevance of the incorporation of realistic microstructures to mesoscopic film growth simulations. In addition, our model incorporates an approach that allows us to reproduce complex microstructures that can develop lattice frustration and texture competition effects.

The rest of the paper is organized as follows. In Sec. II we introduce in detail the numerical model. Section III discusses the results for the $(1+1)$ - and the $(2+1)$-dimensional cases: the different mesoscopic morphologies obtained under different microstructures are analyzed through the introduction of the mean packing density and the mean coordination number. Section IV concludes with a summary of the main results.

\section{MODEL}

\section{A. Particle deposition and microstructures}

In our model we represent the growth units or adatoms as hard disks of radius $a$ whose centers may be located at any position in the continuous space. These units are ballistically deposited on a substrate with periodic boundary conditions at a mean rate $R$. The ballistic trajectories are taken to be normal to the substrate, which is initially configured as a monolayer of fixed adatoms in a close-packed arrangement. Bonds between adatoms are restricted to nearest neighbors. On making the first contact, either with the adatoms on the film surface or directly with the substrate, the incoming particles are instantaneously relocated to the closest available site defined in the microstructure considered. In order to define different microstructures we generalized the off-lattice scheme of Savaloni and Shahraki [28] where instead of locating the adatoms on a fixed lattice, a set of relative positions, the so-called active positions, is defined around each particle of the film as the possible locations at which a new incoming particle can bond. In other words, bonding sites for first nearest neighbors are not fixed with respect to the substrate but are localized around the exposed adatoms. Savaloni and Shahraki introduced this approach to study the complex microstructure formed by the superposition of two elementary bidimensional cells, one generating a simple square and the other corresponding to a hexagonal lattice but the same approach can be used for either simple or composite microstructures. Moreover, while originally the scheme was mainly implemented for films growing transversally to onedimensional substrates giving rise to $(1+1)$-dimensional models, it can be generalized relatively easily to work for two-dimensional substrates also. In this paper we present the results for $(1+1)$ - and $(2+1)$-dimensional systems for different underlying microstructures.

For the $(1+1)$-dimensional case we use four different microstructure combinations shown schematically in Fig. 1: the purely square (sq) and the purely hexagonal (hex) lattices, the hexagonal-square microstructure (hex-sq) - formed by the superposition of the previous ones-and the squaresquare microstructure (sq-sq) obtained by the superposition of two purely square lattices, one horizontal, as the substrate, and the other tilted through $45^{\circ}$. This latter microstructure is a locally defined adaptation of the multilattice scheme from Gilmer, Huang and co-workers for texture competition simulations [31-33]. Both composite microstructures, hex-sq and sq-sq, have mutually exclusive active positions that can produce nonregular arrangements of particles with interesting features such as shadowing of active positions by close offsite adatoms and lattice frustration.

For the $(2+1)$-dimensional system we consider two microstructures: a close-packed hexagonal lattice ( $3 d$-hex) having a maximum coordination number of 12 and a superposition of three pure simple cubic lattices $(3 d$-sc), one following 


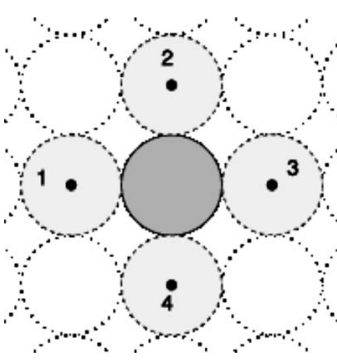

(a)

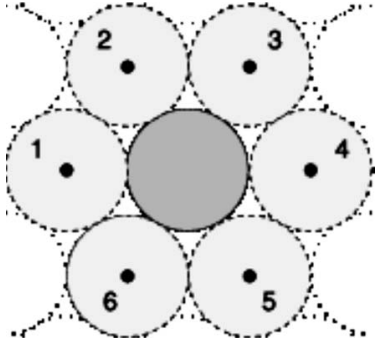

(b)

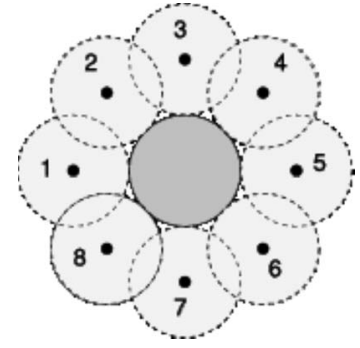

(c)

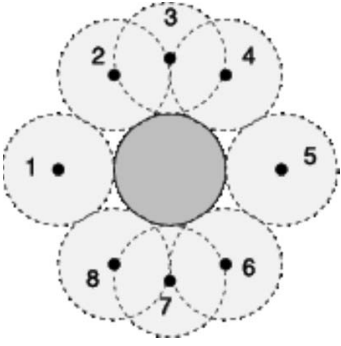

(d)

FIG. 1. Microstructures in the $(1+1)$-dimensional model used in our simulations with their active positions labeled. (a) Simple square lattice with a maximum coordination number 4; a particle is buried when active position 2 is occupied. (b) Simple hexagonal lattice with a maximum coordination 6; the particle is buried when positions 2 and 3 are occupied. (c) Double square lattice with a maximum coordination 4; the particle is buried when positions 2, 3, and 4 are occupied. (d) Two-dimensional projection of the face-centered-cubic lattice (square and hexagonal lattices mixed) with a maximum coordination of 6; a particle is buried when positions 2, 3 , and 4 are occupied.

the $x-y-z$ axis plus another two rotated $45^{\circ}$ around the $x$ axis and $y$ axis, respectively. Its maximum coordination number is 6. Schematic representations of these lattices are shown in Fig. 2 .

In both the $(1+1)$ - and the $(2+1)$-dimensional systems, the sites available for accommodating the incoming particles after their first contact with the film surface at an arbitrary position are the unoccupied and nonshadowed active positions associated with the surrounding surface adatoms. Once a new surface adatom is accommodated, bonds with its nearest neighbors are established and the corresponding unoccupied new active positions are created around it. Figure 3 represents this process schematically.

The evident drawback of this approach for microstructure definition is the increased programming complexity and computational effort in comparison with simple lattice models. In order to achieve computational efficiency a careful implementation of data structures and fast searching algorithms based on binary trees are necessary. The main advantages of our model are a straightforward and flexible definition of simple and complex microstructures within a single framework and the possibility to represent nonregular particle arrangements compatible with the underlying microstructures.
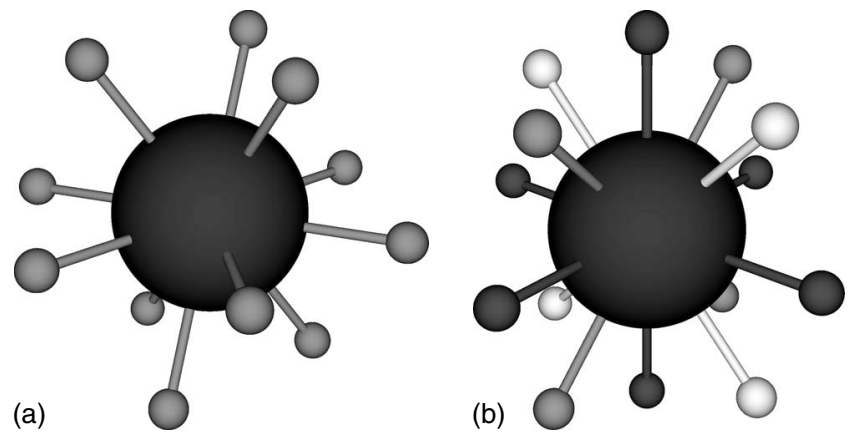

FIG. 2. Three-dimensional microstructures used in the $(2+1)$ simulations. (a) A close-packed hexagonal lattice with a maximum coordination number of 12. (b) A superposition of three pure simple cubic lattices. Its maximum coordination number is 6 . To aid the eye, nearest-neighbor positions lying on the rotated planes are plotted in different gray tones.

\section{B. Surface diffusion: Kinetic Monte Carlo method}

In addition to the process of deposition, surface adatoms are allowed to diffuse thermally to any of the nearby active positions that are neither occupied nor shadowed. Adatoms are considered to belong to the surface when they are not "buried" by the presence of neighbors in the top active positions. In our simulations, diffusive hops are restricted to the nearest neighboring active positions from the original adatom location except for the case of the purely square microstructure in the $(1+1)$ representation. In this case, the relative scarcity and the isotropy of available active positions make quite artificially slow the formation of compact mesoscopic structures induced by nearest-neighbor diffusive hops. Therefore, in order to speed up this process, with a purely square microstructure the adatoms are also allowed to perform diffusive ("over-a-step") hops to second-nearest neighbors.

The superficial diffusion of adatoms is simulated by means of an implementation of the kMC method [34]. Gen-

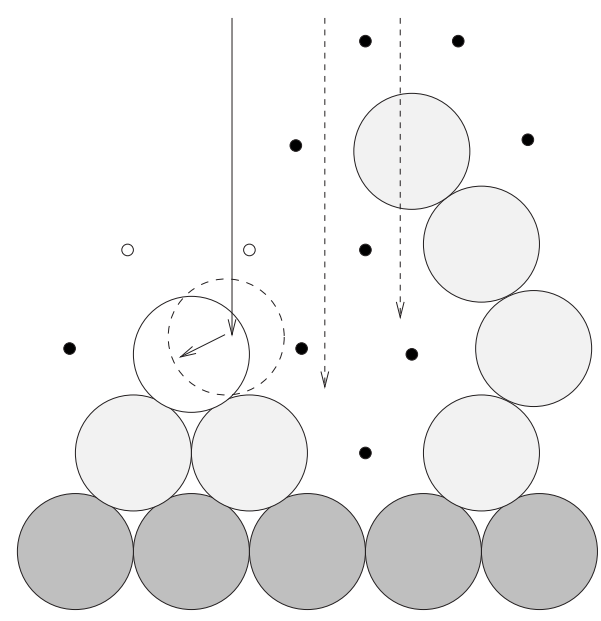

FIG. 3. Illustration of the ballistic deposition process in a simple hexagonal microstructure: fixed substrate (dark circles), shadowed trajectories (dashed arrows), allowed trajectory and instantaneous relaxation (solid arrows) to the closest of the existing active positions (solid dots), and new active positions created around the deposited adatom (open dots). 
erally speaking, kMC methods consist of the evaluation of the transition probabilities between states of a system governed by a list of stochastic events with a given distribution of mean rates and the corresponding realization of the stochastic process. In our case, the list of events is composed of the deposition of a new particle, at a given constant mean rate $R$, together with all the possible diffusive hops of the surface particles, whose mean rates themselves depend on the surface configuration. For the mean rates of the surfacediffusion hops we use the same estimates used by Müller [29] and by Savaloni and Shahraki [28]. Assuming that diffusive hops are activated by thermal fluctuations with frequency $\nu=2 k T / h$, the characteristic mean rate for each possible hop is therefore computed from the Boltzmann statistics which leads to

$$
R_{i \rightarrow j}=\nu \exp \left(-\frac{\Delta E_{i \rightarrow j}}{k T}\right),
$$

where $\Delta E_{i \rightarrow j}$ is the activation energy barrier for the hop between positions $i$ and $j$. Müller estimated this barrier height in terms of the number of bonded neighbors at the origin and destination positions ( $N_{i}$ and $N_{j}$, respectively) as

$$
\Delta E_{i \rightarrow j}= \begin{cases}Q, & N_{i} \leq N_{j} \\ Q+\left(N_{j}-N_{i}\right) \phi, & N_{i}>N_{j},\end{cases}
$$

where $\phi$ is the bonding potential and $Q$ is the surface diffusion activation energy, which is given as a function of the substrate temperature $T$ and the melting point of the material $T_{m}$ by the empirical expression of Neumann and Hirchswald [35]:

$$
Q=\left(5+\frac{20}{3} \frac{T}{T_{m}}\right) k T_{m} \quad\left(T<0.5 T_{m}\right) .
$$

In our simulations no special expression has been used for the step-edge barriers (also known as Ehrlich-Schwoebel (ES) barriers [36,37]).

At each simulation step we compose the list of the rates for the $M$ possible dynamical events of the system, that is, the mean rates of the $M-1$ possible diffusion hops given by Eq. (1) and the mean deposition rate $R$. Then, one event $m$ is randomly selected from the list, with a probability given by its relative mean rate, through the condition

$$
\frac{\sum_{i=0}^{m-1} R_{i}}{\sum_{i=1}^{M} R_{i}}<r<\frac{\sum_{i=1}^{m} R_{i}}{\sum_{i=1}^{M} R_{i}} \quad\left(R_{0}=0\right),
$$

where $r$ is a flat random number in the interval $(0,1)$. After the event is completed, the events list is updated according to the new configuration of the system and the dynamical time step is increased by

$$
\delta t=-\ln \left(r^{\prime}\right)\left[\sum_{i=1}^{M} R_{i}\right]^{-1},
$$

where $r^{\prime}$ is another flat random number in the interval $(0,1)$. For the sake of comparison with the previous results in Ref.

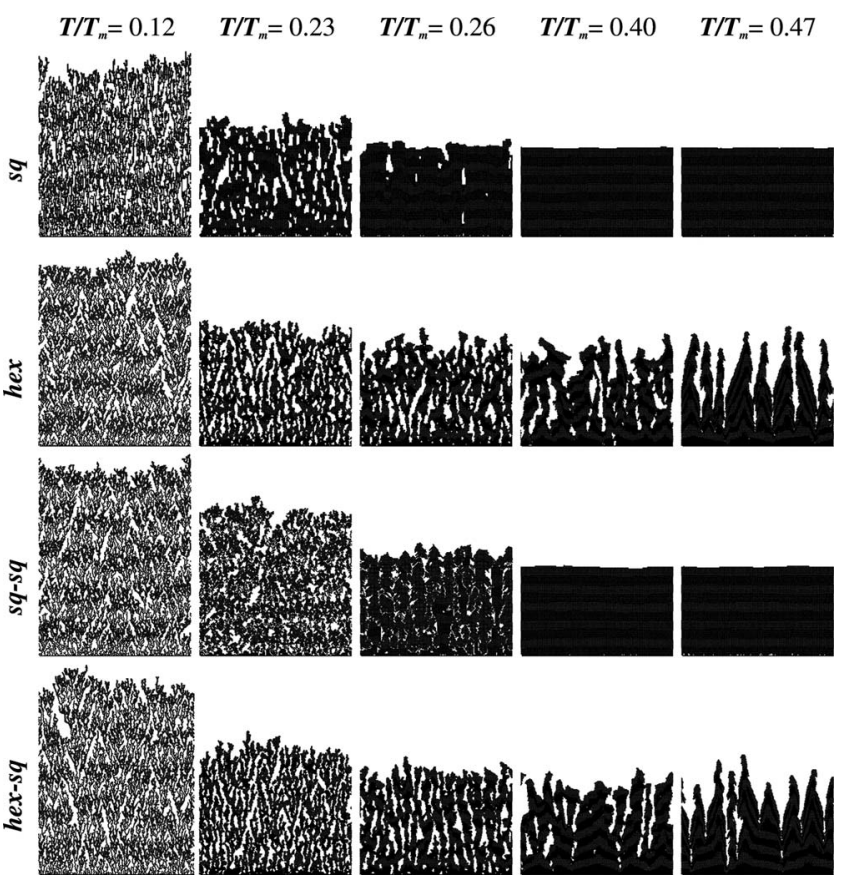

FIG. 4. Film structures obtained at different temperatures at a fixed deposition rate of 1 monolayer per second for each given microstructure; to aid the eye, each stripe represents a height of 10 monolayers.

[28], we picked the same physical parameters corresponding to nickel: $T_{m}=1720 \mathrm{~K}, \phi=-0.74 \mathrm{eV}$, and $a=0.352 \mathrm{~nm}$.

As a final remark on the realization of our simulations, we should point out that the optimization of the searching and updating operations in the list of event rates is essential to the development of fast kinetic Monte Carlo algorithms. To achieve this goal we have adopted an $N$-level binary tree optimization technique following an approach described by Blue et al. [38].

\section{RESULTS AND DISCUSSION}

\section{A. Mesoscopic morphologies}

In the simulations of the $(1+1)$-dimensional model we deposited 15000 particles onto a flat (straight) substrate initially prepared by arranging 160 adatoms in a close-packed configuration. For the type of interactions considered here, this system size is large enough to preclude significant finitesize effects. As we vary the substrate temperature-one of the two most important deposition parameters-we observe the development of different characteristic mesoscopic film morphologies. In Fig. 4 we show the sequences of film morphologies that emerged for each microscopic symmetry combination considered at the same sequence of different substrate temperatures and for a fixed deposition rate $R$ of one monolayer per second $(\mathrm{ML} / \mathrm{s})$. In each case there is a lowest substrate temperature below which low-density dendritic structures are always obtained. These structures are characterized by the self-affine surface geometry typically observed in purely ballistic deposition models without surface diffusion. As the substrate temperature increases, or equivalently 
as deposition rate decreases, surface diffusion becomes significant and dendritic morphologies give way to more compact fibrous structures growing along a preferential axis parallel to the deposition trajectories. With even greater surface diffusion, the former fibrous structures become compact giving rise to densely packed grains that grow vertically as competing columns whose thickness increases with the diffusion. This behavior is qualitatively independent of the underlying microstructure and characterizes the transition from the typical morphologies of the zone I (which are porous and fractallike) to those of the zone II (where pronounced faceting develops until the appearance of structures limited by smooth surfaces) described in the context of the SZM [2,24]. We should emphasize here that the fact that our model does not include any other significant parameters to describe grain boundary evolutions than the underlying microstructure and the competition between deposition rates and surface diffusion makes it not suitable to investigate other zones of the SZM where phenomena such as activated diffusion by ion bombardment of the surface, bulk diffusion, or the presence of impurities are believed to be determinant [2].

In Fig. 4 we can observe the increase in column width as the magnitude of surface diffusion increases for each of the cases considered. However, in spite of this qualitatively universal character, the sequence of emergent mesoscopic morphologies as the deposition parameters are varied seems to be significantly influenced by the underlying microstructure. Thus, with microstructures with a maximum coordination number of 4 such as the simple square (sq) and the squaresquare (sq-sq), we found that the column width eventually reaches the system size. As a consequence, the films form a single compact structure without interstitial voids. Remarkably, this is not the case, at least for the range of temperatures studied, for the (hex) and (hex-sq) microstructures which have a maximum coordination number of 6 . The emergence of such morphological instabilities during epitaxial growth has been associated to the ES effect [39]: during the crystal growth terraces of atomic height are formed. The presence of a step-edge barrier, the so-called ES barrier, hinders the interlayer diffusion preventing atoms landing on these terraces from stepping down. Since grooves cannot easily be filled up, towers of particles form as the ones generated in the (hex) and (hex-sq) microstructures (Fig. 4). Although, as it has been said in Sec. II B, neither step-edge barriers nor asymmetries in the sticking process [40] are explicitly present in our model, the observed behavior can be intuitively well understood. For the simple cubic microstructure (sq) hops to next-nearest-neighbor positions are allowed. The larger number of available active sites with respect to the other microstructures leads to a much easier diffusion around the corners and no ES effect is expected. As a consequence, mounds do not form nor are interstitial voids present in the structure. The most interesting case comes from the comparison between the (hex-sq) and (sq-sq) microstructures. Both share the same amount of active sites and corners [see Figs. 1(d) and 1(c)]; however, the corners of the (hex-sq) microstructure are stable under epitaxial growth onto a horizontal substrate, whereas the corners of the (sq-sq) microstructure are incompatible with the orientation of the substrate. Thus, particles placed on the corners of the (sq-sq)

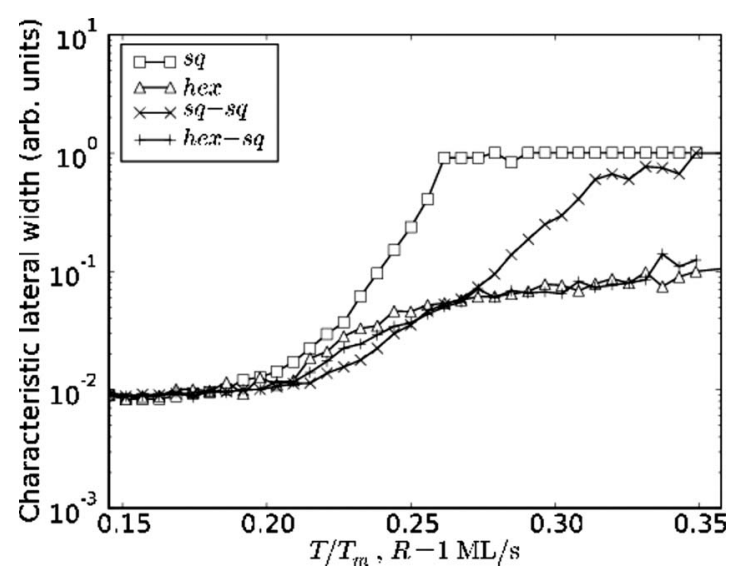

FIG. 5. Change in the mean column width (measured as a fraction of the total width of the substrate) of dense structures with the substrate temperature.

easily step down and no ES effect is observed. On the contrary, particles placed on the corners of the (hex-sq) already occupy a position in the growing structure that is compatible with the substrate orientation giving rise to a virtual ES barrier. The (hex) microstructure behaves similarly to the (hexsq). Finally, the number of stable active sites with respect to the substrate orientation is directly related to the microstructure's maximum coordination number. This effect can be further illustrated through the measurement of the average lateral width of dense structures as a function of the substrate temperature as shown in Fig. 5. This quantity has been sampled by averaging the horizontal lengths at the central regions of the films with a local mean packing density above 0.75 of the maximal density allowed by the microstructure. At high temperatures, the characteristic widths for hex and hex-sq microstructures are very similar and lower than those observed for the sq and sq-sq microstructures. It is also noticeable that the single-square microstructure makes use of the diffusion hops to second-nearest neighbors to reduce the interstitial voids and to increase the characteristic columnar width.

In the simulations of the $(2+1)$-dimensional model we deposited 50000 particles onto a square flat substrate containing $40 \times 40$ adatoms in a close-packed arrangement with periodic boundary conditions in both the $x$ and $y$ directions. In Fig. 6 we show two sequences of film morphologies for the superposition of three simple cubic lattices and the closepacked hexagonal microstructures, respectively, at three different substrate temperatures, $T / T_{m}=0,20,0,26$, and 0,32 and at a fixed deposition rate $R=1$ monolayer per second. As previously observed in the $(1+1)$-dimensional case, we find low-density dendritic structures at temperatures at which surface diffusion is negligible. As the substrate temperature increases, diffusion becomes significant and the film tends to form more compact fibrous structures. From a qualitative observation of the three-dimensional structures shown in Fig. 6 , we can conclude that for those microstructures with a smaller value of the maximum coordination number-the superposition of simple cubic lattices has a maximum coordination number of 6 , whereas for the close-packed hexagonal lattice it is 12-and strong surface diffusion, the films tend to 


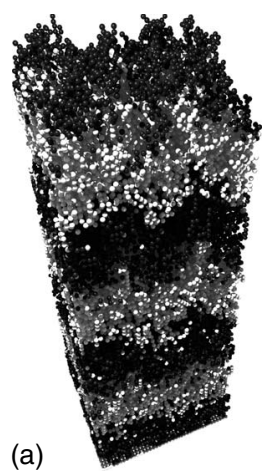

(b)
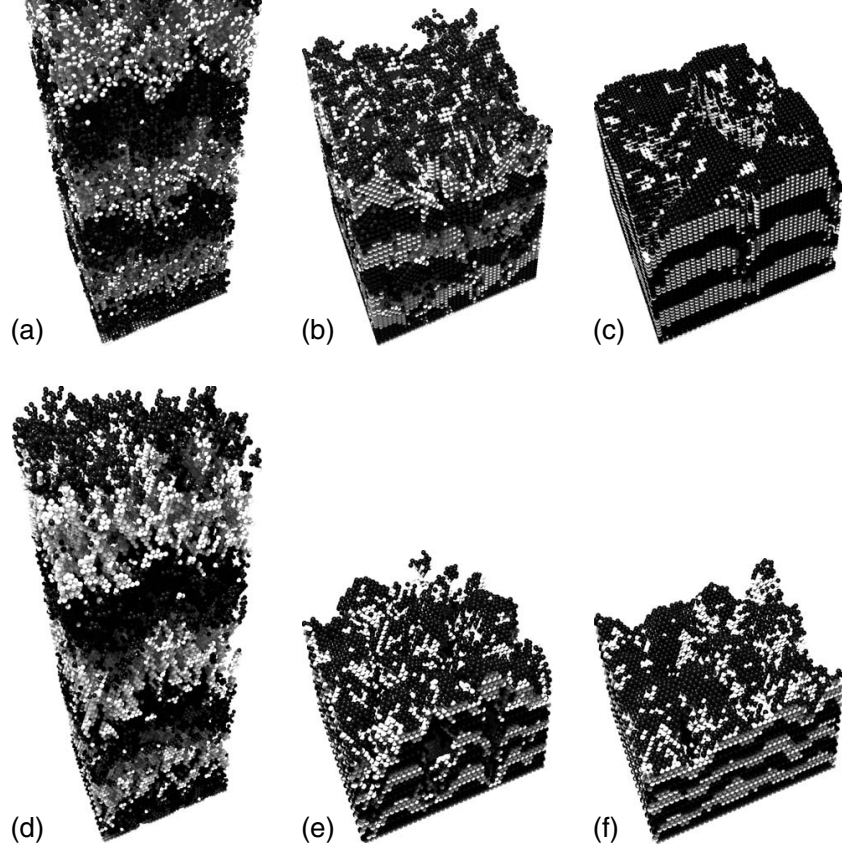

(e)
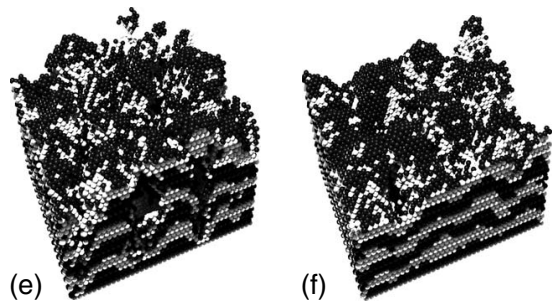

FIG. 6. Three-dimensional film structures obtained after the deposition of 50000 particles at a fixed deposition rate $R$ $=1 \mathrm{ML} / \mathrm{s}$. To aid the eye, layers containing 8000 deposited particles are plotted in alternated dark-light gray tones. Top row: superposition of three simple cubic lattice microstructure $(3 d-\mathrm{sc})$ at reduced temperatures $T / T_{m}$ : (a) 0,20; (b) 0,26; (c) 0,32. Bottom row: close-packed hexagonal microstructure $(3 d$-hex) at reduced temperatures $T / T_{m}$ : (d) 0,20 ; (e) 0,26 ; (f) 0,32 .

form single compact structures without interstitial voids characterized by a rather smooth surface; see Fig. 6(c). On the contrary, for the close-packed hexagonal microstructure under the same deposition conditions, we observe the formation of densely packed grains growing vertically as competing columns; see Fig. 6(f). These results are in agreement with our previous observations for the $(1+1)$-dimensional system.

\section{B. Mean packing density}

The mean packing density is a property frequently used to characterize the evolution of film morphology [28-30]. In simple atomistic simulations the value of the mean packing density increases with the surface diffusion following a sigmoidal law. This sigmoidal behavior, when related to any statistical magnitude that characterizes the particle arrangements in the film, is commonly used to establish the borders between different structure zones. However, the way in which different authors estimate the position of these borders is not consistent. Müller [29] defined a characteristic temperature that fixes the limit between zones I and II at the inflexion point of the curve. On the other hand Savaloni and Shahraki [28] claimed that zones I, II, and III correspond to the segments of the sigmoidal curve characterized by different slopes. Since our model is restricted to structure zones I
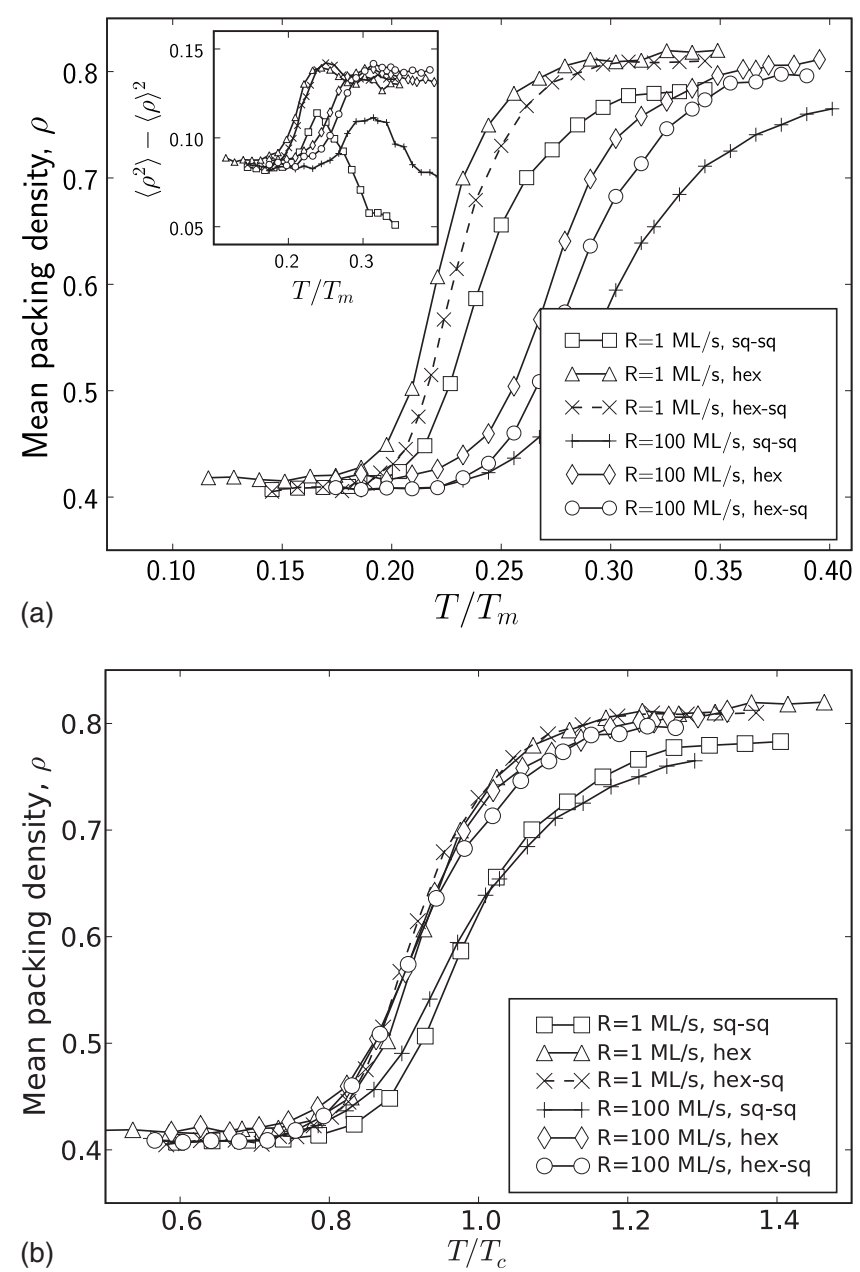

FIG. 7. (a) Change in the bulk mean packing density $\rho$ with the substrate temperature $T$ obtained for different microstructures and at deposition rates $R=1,100 \mathrm{ML} / \mathrm{s}$. The location of the maxima of their statistical fluctuations (inset) determines the characteristic temperature of each curve, $T_{C}$. (b) Mean packing density curves rescaled with the characteristic transition temperature $T_{C}$. Curves corresponding to microstructures sharing the same maximum coordination number collapse onto a single master curve.

and II, we are closer to Müller's interpretation but in order to establish a consistent criterion to identify the structure zone limits, we perform a detailed examination of the statistical fluctuations along the transition curves.

In Fig. 7(a) we plot the variation in the mean packing density $\rho$ with the substrate temperature for the $(1+1)$-dimensional system for three different microstructures and at two different deposition rates, $R=1$ and $R$ $=100 \mathrm{ML} / \mathrm{s}$. This quantity has been computed by sampling the fraction of occupied volume in small regions around each adatom over the whole film structure excluding the substrate and the surface layer. The insets of the same figure show the plot of computed statistical fluctuations of the same quantity. For all the microstructures and deposition rates studied, we found a maximum in the statistical fluctuations of the mean packing density that can be used to identify a characteristic transition temperature as the border between zone I and zone II in the SZM. After normalizing the mean packing density 


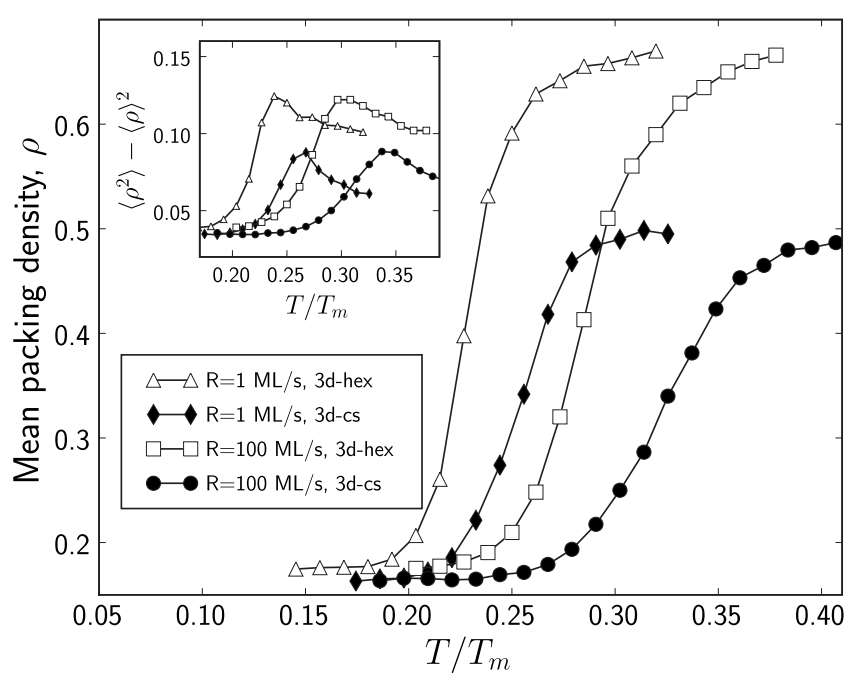

FIG. 8. The same as Fig. 7(a) but for the $(2+1)$-dimensional system. The location of the maxima of the statistical fluctuations (inset) determines the characteristic temperature of each curve, $T_{C}$.

curves with their characteristic transition temperature, $T_{C}$, we observe that all the curves corresponding to the microstructures that share the same maximal coordination number collapse onto a single master curve. Since the maximal coordination number is 4 for the sq and sq-sq microstructures and is 6 for the hex and hex-sq ones, we find the two sets of curves displayed in Fig. 7(b). This result, which is in agreement with the morphological changes in the film structures obtained (Fig. 4) and with the change in the mean column width of the dense structures (Fig. 5), is also a clear indication of the relevance of the underlying microstructure to the structural transitions in solid film growth. A similar behavior is also found with the $(2+1)$-dimensional model. The change in the mean packing density and its statistical fluctuations for the $(2+1)$-dimensional case are displayed in Fig. 8. The sigmoidal curves rescaled with the characteristic temperatures $T_{C}$ at which the fluctuations are maximal are shown in Fig. 9. As for the $(1+1)$-dimensional system we also observe here that curves corresponding to microstructures with the same maximum coordination number -6 for $3 d$-sc and 12 for $3 d$-hex-collapse onto a single master curve. This is again a clear indication that the structural changes at the mesoscopic level are influenced by the value of the maximal coordination number of the microstructure.

The summary of the characteristic transition temperatures in the $(1+1)$-dimensional system, for all microstructures and for deposition rates ranging from $R=10^{-2}$ to $10^{3} \mathrm{ML} / \mathrm{s}$, is shown in Fig. 10. The main difference observed in the behavior of the purely square microstructure sq with respect to the others can be attributed to the hops to second-nearest active positions allowed in this case and resulting in a drastic reduction in the transition temperature.

\section{Mean coordination number}

The previous discussions suggest that the mean coordination number is an appropriate parameter to characterize the structural transitions in solid film growth. We have computed

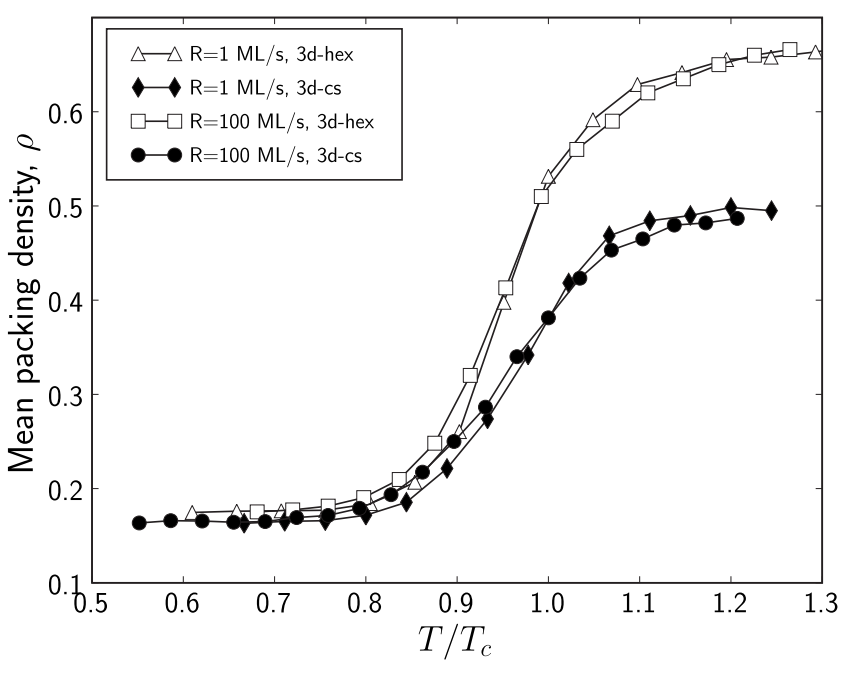

FIG. 9. The same as Fig. 7(b) but for the $(2+1)$-dimensional system. Curves corresponding to microstructures sharing the same maximal coordination number collapse onto a single master curve.

the mean coordination number for the $(1+1)$-dimensional system by averaging the number of bonds per adatom in two different ways: including all the adatoms present in the sample (total values) or including the adatoms located only in the bulk region (bulk values). In Fig. 11 we show the results of these measurements as a function of the substrate temperature. Both the total and the bulk mean coordination numbers change from relatively small values, at the low substrate temperatures that allow porous dendritic structures to develop, to the maximal values allowed by each underlying microstructures at high temperatures. In particular, for the sq and sq-sq microstructures, for which the film eventually forms a single compact shape at high $T$, the mean coordination number reaches its maximum value of 4 ; whereas for the hex and hex-sq microstructures, characterized by the forma-

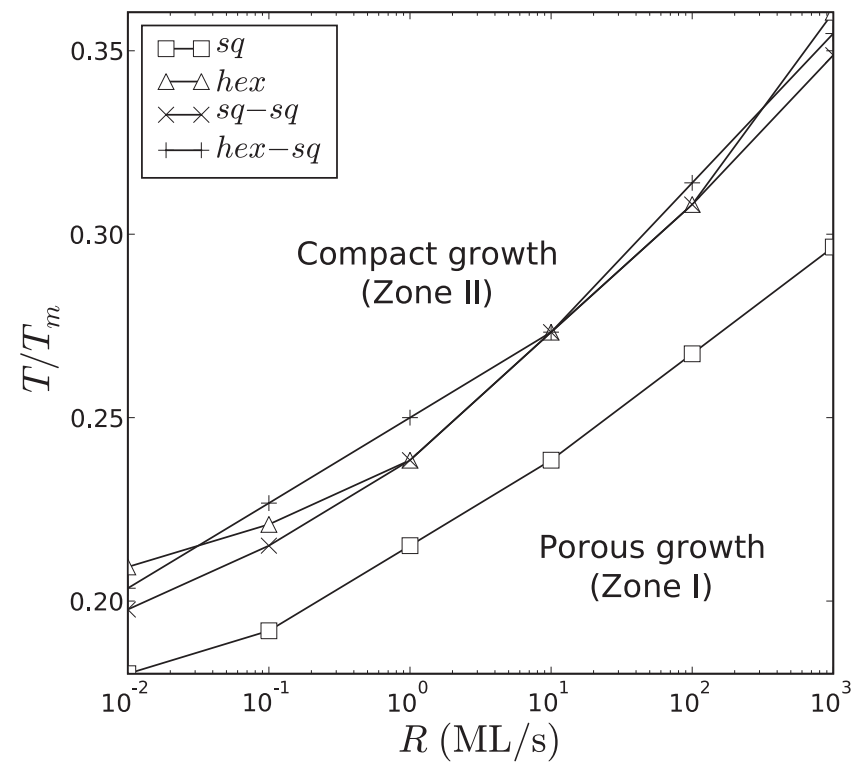

FIG. 10. Change in the transition temperatures between zones I and II, rescaled with the substrate melting temperature $T_{m}$, for each microstructure and at different deposition rates $R$. 

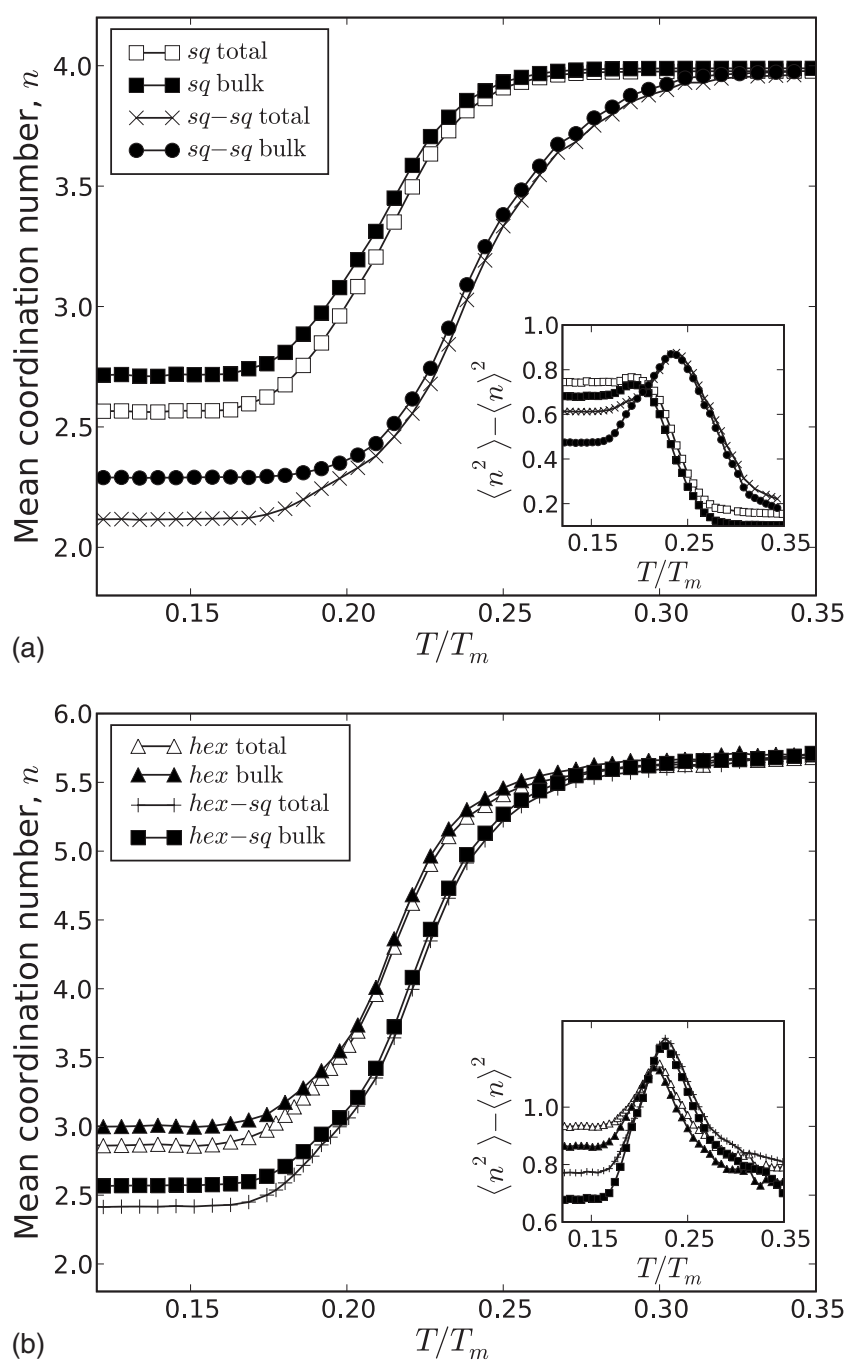

FIG. 11. Total and bulk mean coordination number as a function of the substrate temperature for a deposition rate $R=1 \mathrm{ML} / \mathrm{s}$ and their statistical fluctuations (inset): (a) sq and sq-sq microstructures; (b) hex and hex-sq microstructures.

tion of competing columns at high substrate temperatures, the maximum value achieved by the mean coordination number is slightly below its maximum theoretical value of 6 . All these curves have a rather more complex shape and are more sensitive to the different microstructures than the similar curves obtained for the mean packing density. The existence of a small range of temperatures below the characteristic transitional temperature where the mean coordination number seems to increase linearly is particularly remarkable. The location of the maximum of the corresponding statistical fluctuations (see insets of Fig. 11), that we use to identify a characteristic temperature, is similar for the bulk and total values. These values are slightly lower than the characteristic temperatures derived from the analysis of the mean packing densities, and range from $T / T_{m} \approx 0.19$ to 0.24 for the different microstructures considered. As one might expect, the bulk values of the mean coordination number are higher than the total values for every substrate temperature, but it is noticeable that the differences are considerably larger at low temperatures where surface diffusion is weak or negligible.
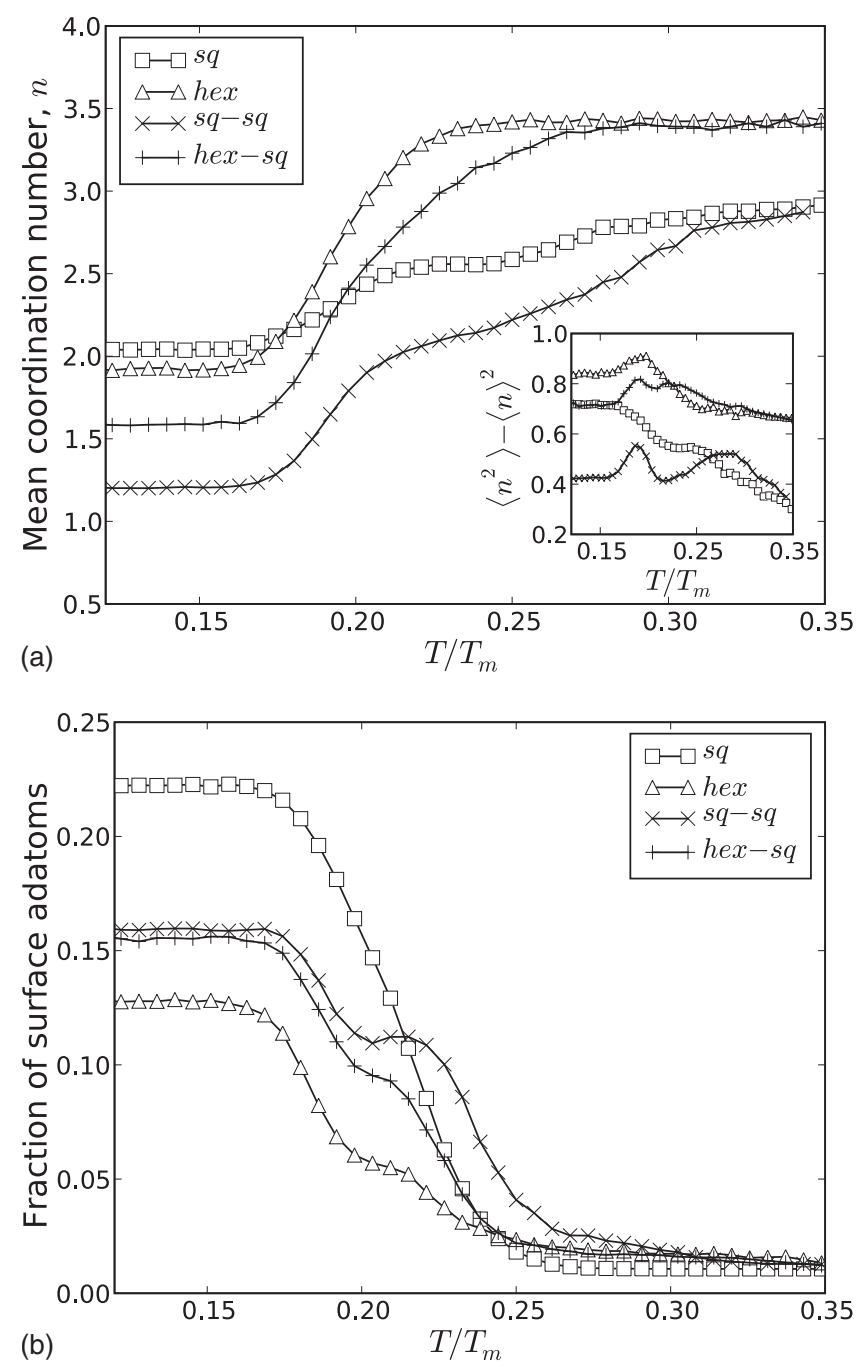

FIG. 12. Statistics for the surface adatoms: (a) mean coordination number and its statistical fluctuations (inset) versus the substrate temperature at a deposition rate $R=1 \mathrm{ML} / \mathrm{s}$ for the different microstructures; (b) total number of surface adatoms under the same conditions.

The complex behavior observed in the mean coordination number can be better understood if we analyze the mean coordination number associated with the surface adatoms. The result for a deposition rate of $R=1 \mathrm{ML} / \mathrm{s}$ is plotted in Fig. 12(a). We can observe that the mean coordination number of surface adatoms grows with the substrate temperature in a way completely different to the bulk and total mean coordination numbers exhibiting a diverse behavior with an apparent strong dependence on the microstructure. By examining the statistical fluctuations [inset in Fig. 12(a)] we observe that there is no clear maximum for the sq microstructure, a single peak for the hex one at $T / T_{m} \approx 0.20$, and a double peak for the sq-sq (at $T / T_{m} \approx 0.19$ and $T / T_{m} \approx 0.28$ ) and the hex-sq (at $T / T_{m} \approx 0.19$ and $T / T_{m} \approx 0.22$ ) microstructures. The location of the characteristic temperatures given by these maxima, considering the first peak when double peaks are present, is, in every case, below characteristic temperatures corresponding to bulk and total values. This fact can be interpreted as if the surface adatoms undergo the tran- 


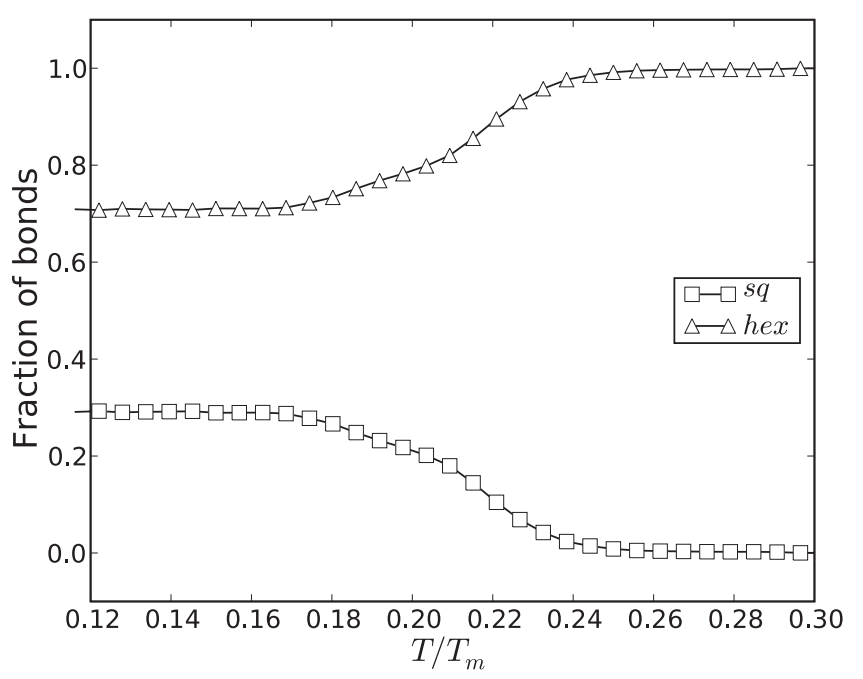

FIG. 13. Fraction of bonds in the hex-sq microstructure as a function of the substrate temperature where symmetries with a maximum coordination number compete. Deposition rate: $R$ $=1 \mathrm{ML} / \mathrm{s}$.

sition in anticipation of the one that occurs in the bulk region.

In addition to this intrinsically complex behavior, the contribution of the surface adatoms to the averaged coordination number is not constant along the transition curves as is shown in Fig. 12(b). The number of surface adatoms decreases with substrate temperature as a result of the film compaction. Interestingly, for all the microstructures except the sq one, a nonmonotonic decrease can be observed suggesting the existence of a double transition consistent with the double peaks observed in the statistical fluctuations of the mean coordination number.

\section{Microstructure competition}

The role of the microstructure is also particularly interesting in the cases where mutually exclusive lattices coexist, as is the case in the sq-sq and hex-sq microstructures. Since microconfigurations of adatoms having larger coordination numbers are energetically more favorable, different competing structures might develop upon the coexistent local symmetries. We can differentiate the two following cases.

\section{Coexistent symmetries with different maximum coordination number: The hex-sq microstructure}

When coexistent lattice symmetries have a different maximum coordination number, as is the case for the composite hex-sq microstructure, the deposited particles in the growing film tend to accommodate according to the lattice symmetry with the higher maximum coordination number, in this case, the hex microstructure. This behavior is illustrated in Fig. 13, where we plot the fraction of bonds in the square and hexagonal lattice symmetries as a function of the substrate temperature. In the strongly diffusive growth regime the deposited particles are finally distributed only in a hexagonal arrangement.

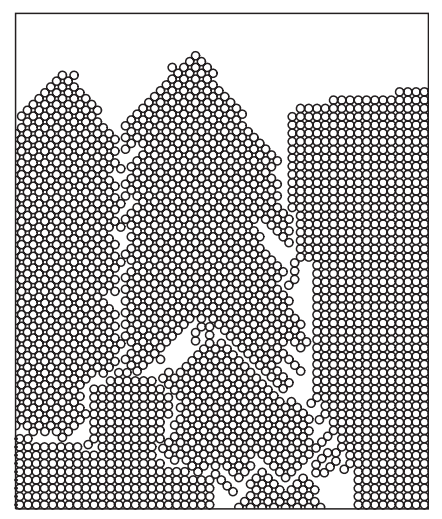

(a)
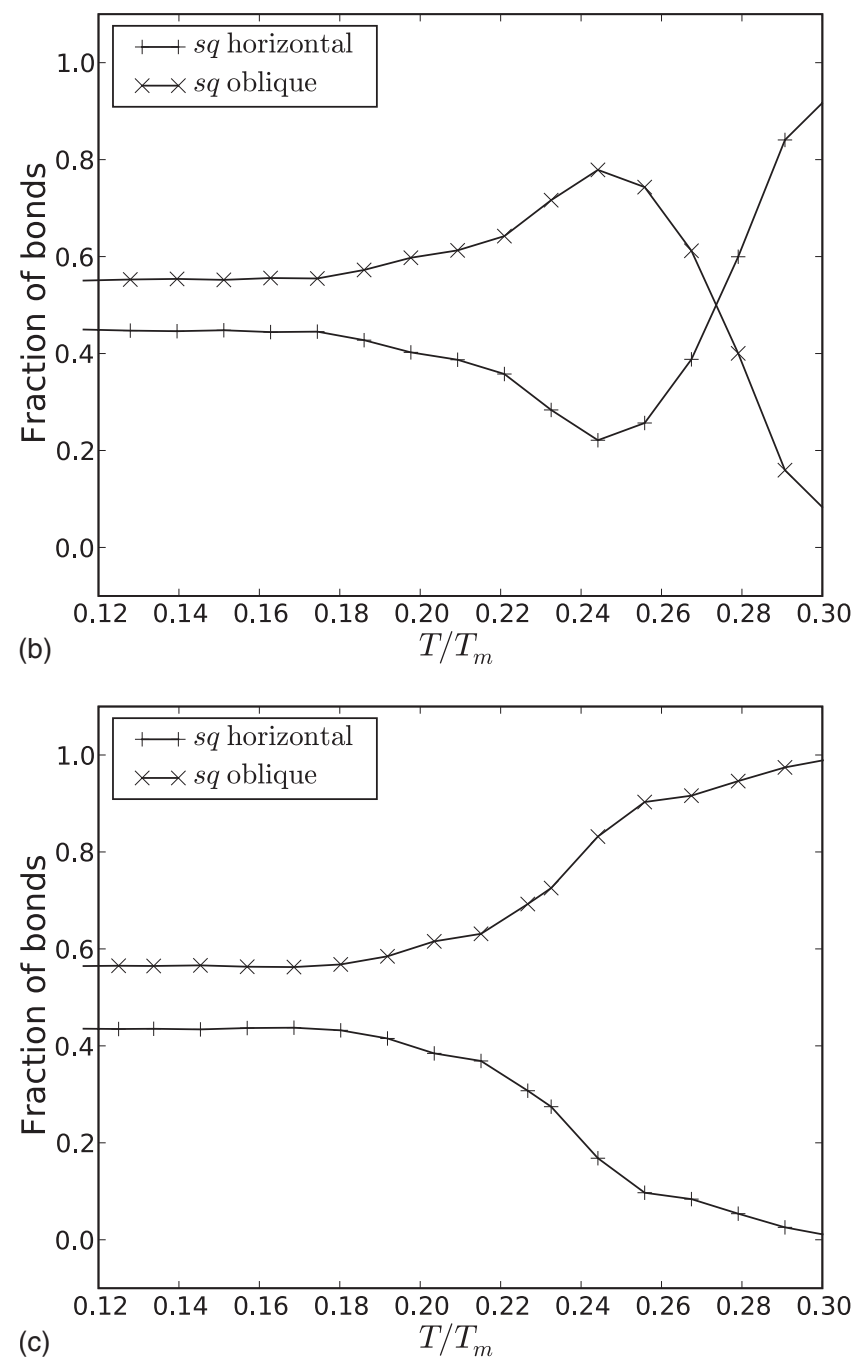

FIG. 14. Texture competition in sq-sq microstructure. (a) Details of single lattice domains formed at intermediate temperatures $(R$ $=1 \mathrm{ML} / \mathrm{s}, T=500 \mathrm{~K})$. Fraction of bonds in the horizontal and the oblique bonding symmetry as a function of the substrate temperature using (b) a horizontal substrate and (c) an oblique substrate.

\section{Coexistent symmetries with the same maximum coordination number: The sq-sq microstructure}

In the sq-sq microstructure, there is no energetically selective mechanism to discriminate between the two compact 
configurations allowed by the underlying composite symmetries. As a result, a polycrystalline film may develop as is illustrated in Fig. 14(a). However, as the surface diffusion strengthens, competing homogeneous growth takes place with the final extinction of one of the symmetries [see Figs. 14(b) and 14(c)]. At relatively low temperatures, diffusion favors the initially more populated symmetry, but when the temperature is high enough the growth reaches the epitaxial regime (well above the characteristic transitional temperature) where the particle arrangement in the film is imposed by the orientation of the bonds in the substrate.

\section{CONCLUSIONS}

We have investigated the influence of competing microstructure symmetries on the emergence of different mesoscopic morphologies in the simulated growth of thin solid films by vapor deposition modeled as $(1+1)$ - and $(2+1)$-dimensional systems. We have used a kinetic Monte Carlo method to model the surface diffusion in combination with a ballistic algorithm adapted to include different microstructure symmetries to model the deposition process. We have focused on the characterization of the transitional structures near the zone boundaries of the empirical SZM.

Our work strongly suggests that the microstructure at the molecular level can have important quantitative influences on the development of film morphologies. For each microstructure considered, the increase in the surface diffusion and the consequent reduction in the shadowing effect as the substrate temperature increases give rise to structures that go from an extremely porous fractal-like pattern, corresponding to zone I in the SZM, to a well defined columnar faceted structure limited by a smooth surface, corresponding to zone II. However, the characteristic temperature at which the transition from zone I to zone II takes place clearly depends on the number of active positions compatible with the substrate orientation that is reflected via the maximum coordination number of the microstructure. For example, as Fig. 4 suggests, higher values of the maximum coordination number lead more easily to frozen fractal structures, so that higher substrate temperatures are required to reach zone II. This behavior can be attributed to the emergence of virtual Ehrlich-Schwoebel barriers that hinder the diffusion around the corners. We have computed the mean packing density as a function of the substrate temperature and find that this quantity is an appropriate parameter to characterize the film morphology. Remarkably, when rescaled according to the characteristic transition temperature derived from the maximum of the density packing fluctuations, this function is found to collapse onto different master curves associated with microstructures sharing the same maximum coordination number.

We also find that the mean coordination number is a more sensitive parameter than the mean packing density, having a more complex behavior close to the characteristic transitional temperatures. In particular, from the analysis of the bulk and surface mean coordination numbers, we can conclude that surface adatoms anticipate (temperature-wise) the transition to be undergone by the adatoms located in the bulk of the film. Furthermore, in those microstructures where mutually exclusive lattice symmetries coexist, a double transition peak appears for the surface adatoms, each associated with its respective symmetry. In addition, competitive morphologies can develop leading to the formation of polycrystalline films at low temperatures. Initially, surface diffusion favors the more populated symmetry but, above the characteristic transitional temperature, epitaxial effects dominate the texture selection.

As a final remark, our simulations also suggest the possible existence of a type of nonequilibrium continuous phase transition in the development of different film morphologies in analogy to those observed in other many-particle nonequilibrium systems such as the directed percolation model [21]. There, a continuous transition from a frozen absorbed state to an active state is observed in the evolution of the system produced by different values of the control parameter. Although a direct change from one phase to the other is not possible here by changing a parameter, the statistics of the resulting states shows the scaling properties of a phase transition. This analogy should stimulate future work on the characterization of morphological changes in the SZM model as such a type of phase transition.

\section{ACKNOWLEDGMENTS}

We thank Russ Messier for useful conversations. This work was undertaken with the financial support of the CSIC project Hielocris and the MICIIN project COSMICAE under Project No. CGL2008-06245-C02-02/BTE. T.S. also acknowledges Project No. FIS2007-60327 funded by the Spanish MEC.
[1] M. Ohring, The Materials Science of Thin Films, 2nd ed. (Academic Press, New York, 2001).

[2] A. Lakhtakia and R. F. Messier, Sculptured Thin Films: Nanoengineered Morphology and Optics (SPIE Press, Bellingham, WA, 2005).

[3] A.-L. Barabási and H. E. Stanley, Fractal Concepts in Surface Growth (Cambridge University Press, Cambridge, England, 1995).

[4] P. Meakin, Fractals, Scaling and Growth Far from Equilib- rium (Cambridge University Press, Cambridge, England, 1997).

[5] D. D. Vvedensky, J. Phys.: Condens. Matter 16, R1537 (2004).

[6] H. N. G. Wadley, X. Zhou, R. A. Johnson, and M. Neurock, Prog. Mater. Sci. 46, 329 (2001).

[7] L. Mandreoli, J. Neugebauer, R. Kunert, and E. Scholl, Phys. Rev. B 68, 155429 (2003).

[8] See, for example, A. Pimpinelli and J. Villain, Physics of Crys- 
tal Growth (Cambridge University Press, Cambridge, England, 1998).

[9] A. Ballestad, T. Tiedje, J. H. Schmid, B. J. Ruck, and M. Adamcyk, J. Cryst. Growth 271, 13 (2004).

[10] A. Ballestad and T. Tiedje, Phys. Rev. B 74, 153405 (2006).

[11] N. G. Nakhodkin and A. I. Shaldervan, Thin Solid Films 10, 109 (1972).

[12] A. G. Dirks and H. J. Leamy, Thin Solid Films 47, 219 (1977).

[13] P. Meakin, Phys. Rev. A 38, 994 (1988).

[14] L. Abelmann and C. Lodder, Thin Solid Films 305, 1 (1997).

[15] R. Messier, V. C. Venugopal, and P. D. Sunal, J. Vac. Sci. Technol. A 18, 1538 (2000).

[16] R. Cuerno and L. Vazquez, in Advances in Condensed Matter and Statistical Mechanics, edited by E. Korutcheva and R. Cuerno (Nova Science Publishers, New York, 2004).

[17] M. Pelliccione, T. Karabacak, and T.-M. Lu, Phys. Rev. Lett. 96, 146105 (2006).

[18] F. Family and T. Vicsek, J. Phys. A 18, L75 (1985).

[19] D. Liu and M. Plischke, Phys. Rev. B 38, 4781 (1988).

[20] T. Karabacak, Y.-P. Zhao, G.-C. Wang, and T.-M. Lu, Phys. Rev. B 64, 085323 (2001).

[21] G. Ódor, Rev. Mod. Phys. 76, 663 (2004).

[22] A. Yanguas-Gil, J. Cotrino, A. Barranco, and A. R. GonzalezElipe, Phys. Rev. Lett. 96, 236101 (2006).

[23] E. Katzav, S. F. Edwards, and M. Schwartz, Europhys. Lett. 75, 29 (2006).

[24] B. A. Movchan and A. V. Demchishin, Phys. Met. Metallogr.
28, 83 (1969).

[25] J. A. Thornton, Annu. Rev. Mater. Sci. 7, 239 (1977).

[26] R. Messier, A. P. Giri, and R. A. Roy, J. Vac. Sci. Technol. A 2, 500 (1984).

[27] P. B. Barna and M. Adamik, Thin Solid Films 317, 27 (1998).

[28] H. Savaloni and M. G. Shahraki, Nanotechnology 15, 311 (2004).

[29] K.-H. Müller, J. Appl. Phys. 58, 2573 (1985).

[30] M. J. Brett, J. Mater. Sci. 24, 623 (1989).

[31] G. H. Gilmer, H. Huang, T. D. de la Rubia, J. D. Torre, and F. Baumann, Thin Solid Films 365, 189 (2000).

[32] H. Huang and G. H. Gilmer, J. Comput.-Aided Mater. Des. 7, 203 (2001).

[33] H. Huang and L. Zhou, J. Comput.-Aided Mater. Des. 11, 59 (2005).

[34] A. C. Levi and M. Kotrla, J. Phys.: Condens. Matter 9, 299 (1997).

[35] G. Neumann and W. Hirschwald, Z. Phys. Chem. Abt. B 81, 163 (1972).

[36] R. L. Schwoebel, J. Appl. Phys. 40, 614 (1969).

[37] G. Ehrlich and F. G. Hudda, J. Chem. Phys. 44, 1039 (1966).

[38] J. L. Blue, I. Beichl, and F. Sullivan, Phys. Rev. E 51, R867 (1995).

[39] A nice review on instabilities in crystal growth can be found in P. Politi, G. Grenet, A. Marty, A. Ponchet, and J. Villain, Phys. Rep. 324, 271 (2000).

[40] I. Elkinani and J. Villain, J. Phys. (France) 4, 949 (1994). 